\title{
On the Planck scale and properties of matter
}

\author{
Ari Lehto \\ Physics Foundations Society, Espoo, Finland
}

Email address:

ari.lehto@physicsfoundations.org

\section{To cite this article:}

Ari Lehto. On the Planck Scale and Properties of Matter. International Journal of Astrophysics and Space Science. Special Issue: Quantum Vacuum, Fundamental Arena of the Universe: Models, Applications and Perspectives. Vol. 2, No. 6-1, 2015, pp. 57-65.

doi: $10.11648 /$ j.ijass.s.2014020601.17

\begin{abstract}
Period doubling, or a frequency halving sequence, is a common property of nonlinear dynamical systems. Period can be related to other physical quantities, e.g. length, energy and temperature, which obtain the corresponding doubling/halving behavior. It is found that physical properties of natural phenomena, systems and elementary particles can be derived directly from the Planck time, taken as the fundamental period. Analysis of experimental data suggests that the period doubling process takes place in three and four internal degrees of freedom. It is further found out that long term stability complies with the stability condition of nonlinear dynamical systems. A theory of period doubling in $1 / r$-type nonlinear systems with three and four internal degrees of freedom is presented.
\end{abstract}

Keywords: Period Doubling, Nonlinear Dynamics, Planck Scale, Elementary Charge, Magnetic Moment, Solar System

\section{Introduction}

The objective of this work is to find out whether period doubling, the characteristic property of nonlinear dynamical systems [1,2], can be found in natural phenomena and $1 / r$-nonlinear gravitational and Coulombic systems. Period doubling means, in terms of frequency, that a sequence of frequencies below the fundamental frequency is borne within the system. Using well known physical relations periods and frequencies can be converted into other physical quantities (e.g. energy), which show the period doubling structure, too. In dynamical systems, an attractor is a set of physical properties toward which a system tends to evolve, regardless of the starting conditions of the system [3]. Period is one of these properties.

Period doubling is a real physical phenomenon, which takes place in many nonlinear systems [4,5,6,7,8]. Period doubling also means self-similarity, and a fractal universe is a topic of modern scientific research [9].

If $\tau_{0}$ is the shortest period in the system, then periods $2 \tau_{o}$, $4 \tau_{o}, 8 \tau_{o}, 16 \tau_{o}$ etc. appear during the doubling process. Period doubling can be identified by taking the ratio $R$ of two periods $\tau_{n}$ and $\tau_{o}$. The ratio will become

$$
R=\frac{\tau_{n}}{\tau_{o}}=2^{n}
$$

and the $n$ 'th period is

$$
\tau_{n}=2^{n} \cdot \tau_{o}
$$

In (2) $n$ is a positive integer. Some periods are more stable than others. Periods of the form

$$
\tau_{n}=2^{2^{n}} \cdot \tau_{o}
$$

are especially stable [2].

The connection between the Planck scale and properties of matter is a long standing issue in physics. The problem is in the extreme values of the Planck scale physical quantities. The Planck energy $E_{o} \approx 3 \cdot 10^{22} \mathrm{MeV}$ is far too large for the rest energy of any elementary particle, the Planck length $l_{o} \approx$ $4 \cdot 10^{-35} \mathrm{~m}$ correspondingly far too small for any real object. The Planck time or period $\tau_{o} \approx 10^{-43} \mathrm{~s}$ is likewise extremely short for any real world event. The Planck scale units are defined using natural constants $h, c, G$ and $\varepsilon_{o}$. In this study $h$ is used instead of $h$-bar, because energy is $h /$ period.

A process generating sub-harmonic frequencies would decrease the Planck energy by bringing about well defined longer periods which, in turn, yield lower energy levels and larger structures etc.

The Planck period can be converted into other physical Planck scale quantities, for instance into energy by $E_{0}=h / \tau_{o}$ and length by $l_{0}=c \tau_{o}$. 
Equation (2) can be generalized for the physical quantities $\mathrm{Q}$, which can be obtained from the Planck period $\tau_{o}$ :

$$
Q_{n}=2^{ \pm n} \cdot Q_{o}
$$

where $n$ is the perceived number of doublings/halvings. The integer part of $n$ in (4) depends on the relative magnitudes of the quantities in comparison, but the fractional part of $n$ is the key issue showing how far $n$ is from an integer. To test (4) several $n$-values were calculated for quantities in different physical phenomena and systems $[10,11,12]$. The distribution of the fractional parts of $n$ is plotted in Fig. 1.

For instance the ratio of the rest energy of the electron-positron pair and the Planck energy yields $n=-74.66$. This is plotted as $n .66$. The ratio of the orbital velocity of Saturn and the speed of light yields $n=-14.93$, plotted as $-n .07$ (a little below an integer value).

The fractional parts seem to group around $0,1 / 3$ and $2 / 3$ suggesting that $n$ is an integer divided by three, which means that the calculated ratios are cube roots of an integer power of 2. Rewriting (4) according to this finding yields

$$
Q_{N}=2^{ \pm \frac{N}{3}} \cdot Q_{o}
$$

where $N=3 n$ is an integer. Cubing both sides of (5) yields

$$
Q_{N}{ }^{3}=2^{ \pm N} \cdot Q_{o}{ }^{3}
$$

Equation (6) suggests that the period doubling process is volumetric for all quantities analyzed in Fig. 1. This means that the properties of the phenomena and systems are determined by three internal (orthogonal by definition) degrees of freedom, which can be visualized by a 3-dimensional geometric structure, which is a right parallelepiped.

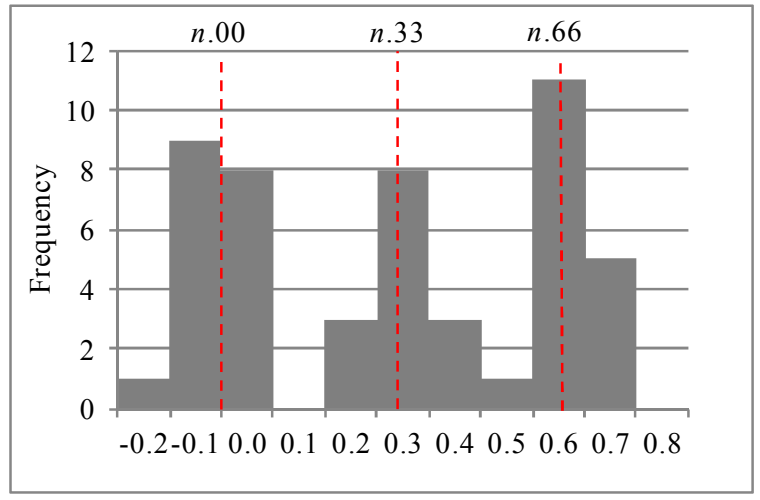

Figure 1. Distribution of fractional parts of $n$ in (4). Bin size is 0.1.

If the periods defining a property (e.g. energy) are $\tau_{i}, \tau_{j}$ and $\tau_{k}$, a 3-d period-volume $V_{\tau}$ is obtained:

$$
V_{\tau}=\tau_{i} \tau_{j} \tau_{k}
$$

where periods $\tau_{i}, \tau_{j}$ and $\tau_{k}$ are the corresponding edge lengths. Assuming that (2) is valid for each of the three periods, then $\tau_{i}=2^{i} \tau_{o}, \tau_{j}=2^{j} \tau_{o}$ and $\tau_{k}=2^{k} \tau_{o}$, and (7) becomes:

$$
V_{\tau}=2^{(i+j+k)} \tau_{o}^{3}
$$

The measured values, or the scalar observables $\tau_{N}$, are obtained by further taking a cube root (=geometric mean) of both sides of (8):

$$
\tau_{N}=2^{\frac{i+j+k}{3}} \tau_{o}=2^{\frac{N}{3}} \tau_{o}
$$

Equation (9) is the same as (5) written in terms of period. $N=i+j+k$ is the total number of period doublings and the volume $V_{\tau}$ in (9) doubles each time any one of the edges doubles.

The corresponding equation for a system with four degrees of freedom is

$$
\tau_{M}=2^{\frac{l+m+n+p}{4}} \tau_{o}=2^{\frac{M}{4}} \tau_{o}
$$

In (9) and (10) $i, j, k, l, m, n$ and $p$ are positive integers. It is important to note that (8) returns a geometric mean of the edge lengths in (7), and thus hides the internal structure of the system. The same applies to (10).

For stable systems $i, j, k$ and $l, m, n, p$ must be powers of 2 themselves according to the stability condition (3). Therefore, it is possible to decompose $N$ into $i, j, k$ and $M$ into $l, m, n, p$ and obtain information about the internal structure of the system.

The ratio of the Coulomb energy of the elementary charge and the Planck charge (Ch. 2.1) obeys (10) indicating that the intrinsic periods related to Coulomb energy double in four degrees of freedom. The elementary particles possess independently both mass-energy $\left(\mathrm{mc}^{2}\right)$ and electric (Coulomb) energy, and therefore (9) and (10) may be combined into one energy-equation:

$$
E_{N, M}=2^{\frac{-N}{3}} \cdot 2^{-\frac{M}{4}} E_{o o}
$$

$E_{o o}$ is the generalized Planck energy. For particles $E_{o o}=2.6388 \cdot 10^{25} \mathrm{MeV}\left(E_{o o}=2^{9.75} \cdot E_{o}\right.$, see Ch. 2.1)

We assume that the intrinsic confined energy can be stored in both rotational and vibrational modes in all degrees of freedom. A rotational mode characterized by period (=circumference) is converted into a vibrational one by division by $\pi$ (circumference to diameter, or circular to linear motion). Multiplication by $\pi$ turns a vibrational mode (diameter) into a rotational one (circumference).

For energy (9) becomes:

$$
E_{N}=\pi^{\frac{n}{3}} 2^{-\frac{N}{3}} \cdot E_{o}
$$

where $n= \pm 1, \pm 2, \pm 3 . n= \pm 1$ means that one of the three degrees of freedom has turned from rotational into vibrational mode or vice versa. For four degrees of freedom (12) becomes 


$$
E_{M}=\pi^{\frac{m}{4}} 2^{-\frac{M}{4}} \cdot E_{o}
$$

where $m= \pm 1, \pm 2, \pm 3, \pm 4$.

Eq. (11) becomes correspondingly

$$
E_{N, M}=\left(\pi^{\frac{n}{3}} 2^{-\frac{N}{3}}\right) \cdot\left(\pi^{\frac{m}{4}} 2^{-\frac{M}{4}}\right) \cdot E_{o o}
$$

In principle $n, N, m$ and $M$ are independent of each other in (14).

\section{Data Analysis}

The gravitational constant $G$ is the main source of inaccuracy in the Planck scale units.

The relative standard uncertainty of $G$ is $120 \mathrm{ppm}$ and hence that of the Planck mass, energy and temperature is 60 $\mathrm{ppm}$. The values of the natural constants have been adopted from the NIST table of natural constants.

The calculated values used for reference are displayed in Table 1.

\subsection{Elementary Electric Charge}

Table 1 shows that the Planck charge $q_{o}=\left(4 \pi \varepsilon_{o} h c\right)^{1 / 2}$ is surprisingly close to the elementary electric charge $(e=$ $1.602 \cdot 10^{-19}$ As) differing only by a factor of about 29 . Obviously equality would be the simplest case, but the difference suggests that sub-charges have been created in the process of time, as the internal period doubling has continued. The ratio of the Coulomb energies (charges squared) is

$$
\frac{q_{o}^{2}}{e^{2}}=2^{m}
$$

Equation (15) yields the perceived number $m$ of period doublings, or energy halvings, needed for the Coulomb energy of the Planck charge to become the Coulomb energy of the elementary electric charge. Calculation yields

$$
\frac{q_{o}^{2}}{e^{2}}=2^{9.7499} \cong 2^{\frac{39}{4}}=2^{\frac{l+m+n+p}{4}}
$$

which (16) means that the total number of period doublings is $M=4 m=39$ in four degrees of freedom.

According to (16), a perceived charge $e^{2}$ is created:

$$
e^{2}=2^{-\frac{39}{4}} \cdot q_{o}^{2}=g \cdot q_{o}^{2}
$$

where $g=2^{-39 / 4}$. The elementary charge squared is superstable (otherwise we would not exist in our present form). It should be now possible to decompose $M=39$ into superstable periods according to (3). Indeed, $M$ can be written as $1+2+4+32=2^{0}+2^{1}+2^{2}+2^{5}$, which shows the superstability (3) of the related periodic structure.

The value of $e$ can be calculated from (17) with $g=2^{-39 / 4}$. One obtains $e=1.60213 \cdot 10^{-19}$ As, which is accurate to $30 \mathrm{ppm}$ compared to the NIST value. Taking the square root of $e^{2}$ in (17) also yields the polarity of the electric charge.

Equation (17) suggests that electrical charges other than the elementary charge may exist. High energy particle collisions may create suitable conditions for a short while (i.e. other $g$-values). This situation would be, in a sense, a time reversal, because the elementary charge would evolve back towards the Planck charge. This situation would confuse the calculation of the elementary particle masses, because the elementary electric charge or its multiple is now assumed for all charged particles. We conclude that the number of degrees of freedom is four for the Coulombic energy systems.

Table 1. Planck scale references

\begin{tabular}{lll}
\hline Quantity & Value & \\
\hline Energy $E_{o}$ & $3.0604 \cdot 10^{22}$ & $\mathrm{MeV}$ \\
Length $l_{o}$ & $4.0512 \cdot 10^{-35}$ & $\mathrm{~m}$ \\
Temperature $T_{o}$ & $3.5515 \cdot 10^{32}$ & $\mathrm{~K}$ \\
Velocity $c$ & 299792458 & $\mathrm{~m} / \mathrm{s}$ \\
Mag. mom-orb $\mu_{o-o r b}$ & $1.5485 \cdot 10^{-46}$ & $\mathrm{Am}^{2}$ \\
Mag. mom-rad $\mu_{o-\text { rad }}$ & $3.8207 \cdot 10^{-46}$ & $\mathrm{Am}^{2}$ \\
Charge $q_{o}$ & $4.7013 \cdot 10^{-18}$ & $\mathrm{As}$ \\
\hline
\end{tabular}

\subsection{Electron}

The observable intrinsic properties of an electron are the rest energy (or mass), the electric charge and the magnetic moment.

\subsubsection{Charge}

The elementary charge is calculated in Ch. 2.1.

\subsubsection{Rest Energy}

The electron-positron pair creation is the lowest energy mass creation process. The electron and the positron always come together forming a particle-antiparticle combination. Comparison of the rest energy $1.022 \mathrm{MeV}$ of the ep-pair and the Planck energy $E_{o}$ yields

$$
\frac{1.022}{E_{o}}=2^{-74.665} \cong 2^{-\frac{224}{3}}=2^{-\frac{i+j+k}{3}}=2^{-\frac{32+64+128}{3}}
$$

where 74.665 is the perceived number of period doublings. Using $N=3 \cdot 74.665=224$ for the total number of doublings one obtains $1.021 \mathrm{MeV}$ for the ep-pair mass, which differs from the NIST value by $0.1 \%$. As can be seen the ep-structure is superstable $\left(N=2^{5}+2^{6}+2^{7}\right)$ as it should be. One more period doubling in all three degrees of freedom $(N=227)$ halves the energy of the pair, and two $0.511 \mathrm{MeV}$ particles are borne. Mass is non-polar, unlike the electric charge, because the rest energy of the ep-pair is obtained by taking a cube root which gives either positive (this case) or negative values.

It is worth noting that it is the ep-pair that is superstable. For this reason it is tempting to think that it is the positron that gives the proton its positive elementary charge.

\subsubsection{Magnetic Moment}

We need to define the Planck scale magnetic moment for calculating the magnetic moment of the ep-pair. Magnetic moment $\mu$ is classically defined as a current loop, where the 
current is $i$ and the loop area $A$. The magnetic moment of the loop is $\mu=i A$. We denote the Planck scale reference by $\mu_{o-o r b}$ meaning that the circumference (orb) of the loop is the Planck length $l_{o}$ in Fig. 2. The loop current is the elementary charge divided by period, i.e. $i=e / \tau_{o}$. The magnetic moment becomes

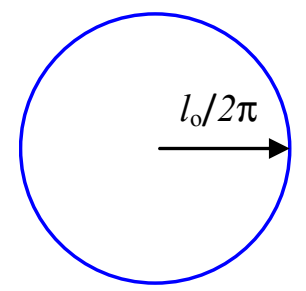

Figure 2. The Planck scale magnetic moment for the electron.

$$
\mu_{o-o r b}=i A=\frac{e}{\tau_{o}}\left(\pi r^{2}\right)=\frac{e c^{2} \tau_{o}}{4 \pi}
$$

The value of $\mu_{o-o r b}$ is $1.5485 \cdot 10^{-46} \mathrm{Am}^{2}$. Equation (19) shows that the magnetic moment grows with growing period contrary to energy, which decreases. For the ep-pair

$$
\frac{1 / 2 \mu_{e}}{\mu_{o_{-} \text {orb }}}=2^{74.6664} \cong 2^{\frac{224}{3}}=2^{\frac{32+64+128}{3}}
$$

The magnetic moment of the electron (with $N=227$ ) is twice that of the ep-pair's magnetic moment $(N=224)$ because the magnetic moment grows with $N$. Note also the same total number of doublings as before for the ep-pair mass, as if mass and magnetic moment evolved together. $\mu_{e}$ in (19) is the measured magnetic moment of the electron $\left(9.2848 \cdot 10^{-24} \mathrm{Am}^{2}\right)$.

The calculated value of $\mu_{e}$ becomes $9.2863 \cdot 10^{-24} \mathrm{Am}^{2}$, which differs from the NIST value by $0.016 \%$.

\subsection{Proton}

The observable intrinsic properties of a proton are the rest energy (or mass), the electric charge and the magnetic moment.

\subsubsection{Charge}

As in Ch. 2.1.

\subsubsection{Rest Energy}

Applying (14) for a proton yields

$$
\frac{E_{p}}{E_{\text {oo }}}=\left(\pi^{\frac{0}{3}} 2^{-63.997}\right) \cdot\left(\pi^{-\frac{2}{4}} 2^{-9.75}\right)
$$

where $m=0, N / 3=63.997, m=-2$ and $M / 4=9.75 . E_{p}=938.28$ $\mathrm{MeV}$ is proton's rest energy. The first part in (21) describes the 3-d mass-energy showing that the three degrees of freedom are all rotational $(n=0$ in (14)). In the second part $m=-2$ in (21) means that two of the four degrees of freedom of the Coulomb energy system are vibrational. $M=39$ means that the number of period doublings of the Coulomb energy is the same for the proton and the electron-positron pair. Eq.
(21) shows that $N / 3=63.997 \cong 64$. As may be expected $N=3 \cdot 64=2^{6}+2^{6}+2^{6}$ is superstable. For $N=3 \cdot 64=192$ the proton mass becomes $936 \mathrm{MeV}$.

Equation (21) shows that the proton alone is superstable, unlike the electron-positron pair, where the pair is superstable. The antiproton does not show up in (21), and therefore some property of the neutron must possess the 'anti'-property.

\subsubsection{Magnetic Moment}

Because the 4-d Coulomb energy of the proton seems to have two vibrational modes, we define the Planck scale magnetic moment in Fig. 3 in such a way that the diameter of the current loop is half of the Planck length corresponding to the lowest energy state of a particle in a box. Definition of the Planck magnetic moment $\mu_{o-r a d}$ (radial type) is:

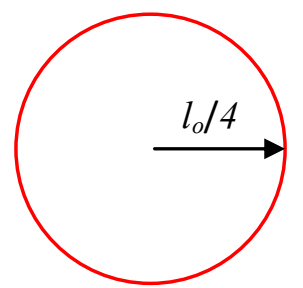

Figure 3. The Planck scale magnetic moment for the proton.

$$
\mu_{o-r a d}=i A=\frac{e}{\tau_{o}}\left(\pi r^{2}\right)=\frac{\pi}{16} e c^{2} \tau_{o}
$$

The value of $\mu_{\text {o-rad }}$ is $3.8207 \cdot 10^{-46} \mathrm{Am}^{2}$ and

$$
\frac{1 / 2 \mu_{p}}{\mu_{o-r a d}}=2^{64.000}
$$

for the nucleon pair. One more period doubling $(N=65)$ doubles the magnetic moment of the pair for each particle. The calculated value of $\mu_{p}$ becomes $1.4096 \cdot 10^{-26} \mathrm{Am}^{2}$, which differs from the NIST value $\left(1.4106 \cdot 10^{-26} \mathrm{Am}^{2}\right)$ by $0.07 \%$.

\subsection{Neutron}

\subsubsection{Charge}

A neutron has a negatively charged surface layer, a positively charged middle layer, and a negative core. The total electric charge is zero.

\subsubsection{Rest Energy}

The rest mass of the neutron is $1.28 \mathrm{MeV}$ larger than that of the proton. This energy corresponds to the first 'excited state' of the ep-pair, since $1.29 \mathrm{MeV}=2^{0.333} \cdot 1.022 \mathrm{MeV}$.

\subsubsection{Magnetic Moment}

The charge distribution suggests that the magnetic moment $\mu_{n}$ of the neutron can be modeled by three current loops. In Fig. $4 \mu_{s}$ is the magnetic moment of the negative surface current loop, $\mu_{m}$ of the positive middle layer loop and $\mu_{c}$ that of the negative core loop. If the area of the negative core loop is small compared to the negative surface loop, then the magnetic moment $\mu_{c}$ of the core can be neglected for the first approximate calculations. 
The proton and the neutron sizes are essentially equal, and we simply assume that the magnitude of the magnetic moment caused by the negative surface is the same as the proton's magnetic moment, but of opposite sign. The magnetic moment $\mu_{m}$ of the middle part is obviously smaller and positive. With these assumptions we can write

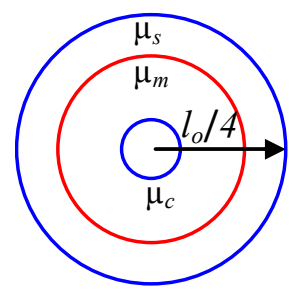

Figure 4. The Planck scale magnetic moment for the neutron according to the charge distribution.

$$
\mu_{\mathrm{n}} \cong-\mu_{\mathrm{p}}+\mu_{\mathrm{m}}
$$

The measured magnetic moments are $\mu_{p}=1.4106 \cdot 10^{-26} \mathrm{Am}^{2}$ and $\mu_{n}=-9.6624 \cdot 10^{-27} \mathrm{Am}^{2}$. The 'middle' magnetic moment $\mu_{m}$ can be solved for from (24) yielding $\mu_{m}=4.438 \cdot 10^{-27} \mathrm{Am}^{2}$. The ratio of $\mu_{m}$ and $\mu_{o-r a d}$ is

$$
\frac{\mu_{m}}{\mu_{o-r a d}}=2^{63.333}=2^{\frac{190}{3}}
$$

showing that the 'middle' magnetic moment belongs to the same period doubling sequence as the proton. Inserting the calculated $\mu_{m}$ into (24) yields $\mu_{n}=-9.662 \cdot 10^{-27} \mathrm{Am}^{2}$ for the neutron, which is practically the same as the measured value. We mentioned earlier that the antiproton does not show up in (21), and therefore some property of the neutron must possess the 'anti'-property. This analysis suggests that the negative surface magnetic moment of the neutron is the required 'anti'-property.

\subsection{Deuteron Magnetic Moment}

A proton and a neutron are together in a deuterium nucleus. We would assume the neutron surface magnetic moment $\mu_{s}$ to cancel the proton magnetic moment $\mu_{p}$ leaving the 'middle' magnetic moment for the deuteron. The measured value of the deuteron magnetic moment is $4.331 \cdot 10^{-27} \mathrm{Am}^{2} . \mu_{m}$ from (24) is nearly the same as the measured value supporting our assumption (2.5\% difference). The close-by positive magnetic moment of the proton and the negative surface magnetic moment of the neutron create an attractive force and magnetic binding.

\section{6 .Fine Structure Constant}

The fine structure constant $\alpha$ is defined as

$$
\alpha^{-1}=\frac{2 \varepsilon_{o} h c}{e^{2}}=\frac{4 \pi \varepsilon_{o} h c}{2 \pi e^{2}}=\frac{1}{2 \pi} \cdot \frac{q_{o}{ }^{2}}{e^{2}}=137.036
$$

Because $e^{2}=2^{-39 / 4} \cdot q_{o}^{2},(26)$ can be written in the form

$$
\alpha=2 \pi g
$$

where $g=2^{-39 / 4}$ is the geometric factor reflecting the different internal structures of the two energy systems $e^{2}$ and $q_{o}{ }^{2}$.

The calculated value (27) of $1 / \alpha=137.0448$ differs from the NIST value by $60 \mathrm{ppm}$.

\subsection{Hydrogen $21 \mathrm{~cm}$ Line}

A neutral hydrogen atom can experience an electron magnetic moment reversal relative to the magnetic moment of the proton. The energy released corresponds to $21.12 \mathrm{~cm}$ wavelength $(5.87 \mu \mathrm{eV})$ and can be observed in a laboratory and astronomical sources. Equation (1) yields

$$
\frac{21.12 \mathrm{~cm}}{l_{o}}=2^{112.006} \cong 2^{\frac{336}{3}}
$$

With $\quad N=3 \cdot 112=336=2^{4}+2^{6}+2^{8}, \quad$ one obtains $\lambda=$ $2^{(16+64+256) / 3} \cdot l_{o}=21.04 \mathrm{~cm}$. If comparison is made to the Compton wavelength $\lambda_{\mathrm{Ce}}\left(1.213 \cdot 10^{-12} \mathrm{~m}\right)$ of the electron-positron pair, one obtains:

$$
\frac{21 \mathrm{~cm}}{\lambda_{C e}}=2^{37.34} \cong 2^{\frac{112}{3}}
$$

Note that both $N=336=2^{4}+2^{6}+2^{8}$ and $N=112=2^{4}+2^{5}+2^{6}$ are superstable. This means that the energy of the magnetic moment reversal of the electron is directly related to the ep-pair rest energy $(N=224)$.

\subsection{Cosmic Background Radiation}

The $3 \mathrm{~K}$ cosmic background radiation (CBR) is detected as microwave photons coming from all over the sky. The spectrum of the radiation corresponds to radiation within a blackbody, whose temperature is $T_{C B R}=2.73 \mathrm{~K} \quad(k T=0.235$ $\mathrm{meV}$ ). Equation (1) yields

$$
\frac{T_{C B R}}{T_{o}}=2^{-106.68} \cong 2^{-\frac{320}{3}}
$$

$T_{o}$ is the Planck temperature. $T_{C B R}=2.76 \mathrm{~K}$ with $N=320=$ $(64,128,128)$, which is superstable.

If comparison of $E_{C B R}=k T$ to the $1.022 \mathrm{MeV}$ gamma photon is made, one obtains

$$
\frac{E_{C B R}}{1.022}=2^{-32.02}
$$

suggesting that $\mathrm{CBR}$ is directly related to the rest energy of the ep-pair.

The number of period doublings links the CBR to the ep-pair and the $21 \mathrm{~cm}$ wavelength. If CBR is considered as the remnant glow of the 'big bang', then the temperature of the 'big bang' has originally been the Planck temperature, now diluted by period doubling. In view of this study it seems more likely that $\mathrm{CBR}$ is a property of the ep-pair 
energy system originating from the Planck energy.

The next colder temperature is given by $N=321=(64,128$, 129), which corresponds to $T=2.19 \mathrm{~K}$. It is interesting to note that this temperature is close to the $2.17 \mathrm{~K} \lambda$-point of liquid helium ${ }^{4} \mathrm{He}$. This is the temperature where helium becomes superfluid and detaches from the Earth reference.

\subsection{The Solar System}

The Solar system can be described as a $1 / r$-nonlinear closed system dominated by the gravitational potential of the Sun. Ideally a planet orbits on an equipotential surface with a constant velocity. The period doubling process would discretize both the orbital velocity and the circumference (and the radius) of the orbit of a planet.

Period doubling yields velocities

$$
v_{N}=2^{-\frac{N}{3}} \cdot c
$$

and circumferences of the orbits

$$
l_{M}=2^{\frac{M}{3}} \cdot l_{o}
$$

Equations (32) and (33) determine the values for the orbital velocities and distances $r_{M}=l_{M} / 2 \pi$ from the Sun in the period doubling condition. These values are fixed, because the Planck scale velocity and length are used as reference. Eq. (32) is derived in [11]. The geometric picture of the system would resemble a Sun-centered onion, where each layer has a specific velocity and radius.

Table 2, plotted in Fig. 5, displays the observed and calculated orbital velocities of the planets (Pluto included).

The vertical axis shows the orbital velocity in $\mathrm{km} / \mathrm{s}$, and the horizontal axis the distance from the Sun in astronomical units (AU). The asteroids $(N=42)$ occupy the gap between Mars and Jupiter. The consequent $M$-values range from $M$ $=38$ (Mercury) to $M=48$ in (32) with one exception. There is an unoccupied allowed orbit with $M=44$ between Jupiter and Saturn, as if a planet were missing there, too.

Table 2. Observed and calculated orbits of the planets

\begin{tabular}{cccccc}
\hline Planet & Obs $\mathbf{~ k m} / \mathbf{s}$ & $\mathbf{N}$ & Calc km/s & Obs AU & Calc AU \\
\hline Mercury & 47.5 & 38 & 46.1 & 0.39 & 0.39 \\
Venus & 35.0 & 39 & 36.6 & 0.72 & 0.78 \\
Earth & 29.8 & 40 & 29.1 & 1.00 & 0.98 \\
Mars & 24.1 & 41 & 23.1 & 1.52 & 1.56 \\
Asteroids ${ }^{1}$ & 18.0 & 42 & 18.3 & 2.77 & 2.47 \\
Jupiter & 13.1 & 43 & 14.5 & 5.19 & 4.95 \\
Missing & N/A & 44 & 11.5 & N/A & 7.85 \\
Saturn & 9.6 & 45 & 9.2 & 9.51 & 9.89 \\
Uranus & 6.8 & 46 & 7.3 & 19.13 & 19.79 \\
Neptune & 5.3 & 47 & 5.8 & 29.98 & 31.41 \\
Pluto & 4.7 & 48 & 4.6 & 39.50 & 39.58 \\
${ }^{1}$ Ceres, Pallas & & & & & \\
\hline
\end{tabular}

Note that the asteroids and the empty $M=44$ orbit are on both sides of Jupiter.

The calculated $(r, v)$ values are denoted by yellow squares, and the planets, denoted by the grey diamonds, seem to stay in the immediate vicinity of the calculated $(r, v)$ - values.

Although the $M$-values for the velocity are consequent integers, not all allowed orbits are occupied, however. It is shown in $[10,11]$ that there are two allowed orbits between Mercury and Venus, one between the Earth and Mars, etc. This is because the orbital velocity hyperbola $v=$ sqrt $\left(G M_{S u n} / r\right)$ does not fulfill both the velocity (32) and circumference (33) conditions, i.e., the hyperbola does not cross, or go sufficiently near a $(r, v)$ fixed-point in order for there to be a mass concentration.

This is a fundamental difference to Kepler's third law, because the fixed points (32) and (33) are independent of the central mass, unlike the $G m M / r$ hyperbola.

The green curve in Fig. 5 shows the $v=\operatorname{sqrt}\left(G M_{S u n} / r\right)$ hyperbola for the mass of the Sun. For another star the hyperbola would be at a different location, and therefore the unoccupied orbits would be different, too (because the hyperbola would cross other fixed points). The orbital velocities would still be given by (32). This is illustrated in Fig. 6 for two different center masses.

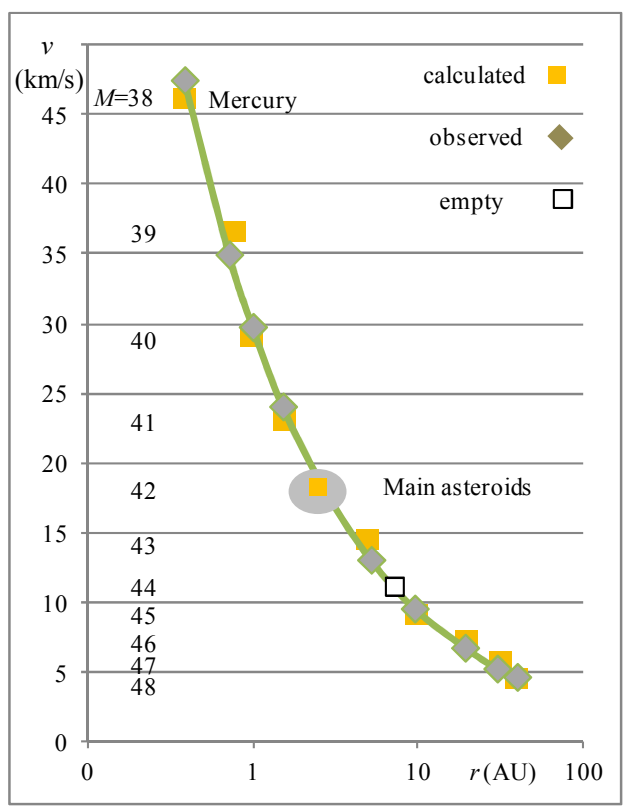

Figure 5. Comparison of the observed and calculated orbits.

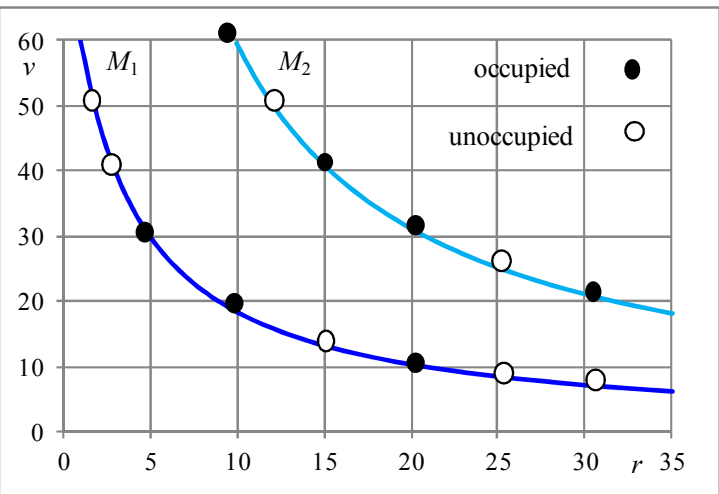

Figure 6. Location of the occupied and unoccupied orbits depends on the magnitude of the center mass. The horizontal lines denote the fixed velocities (32) and the vertical lines the fixed distances (33). 
The distances $r_{M}$ of the planets from the Sun obey the so-called Titius-Bode rule, found experimentally (not valid beyond Uranus). One form of the rule is $r_{M}=r+0.4$ in astronomical units with $r=0,0.3,0.6,1.2,2.4, \ldots$ (doubling $r, 0$ for Mercury).

The difference between the Titius-Bode rule for distances and $(33)$ is that the $(r, v)$ quantization by period doubling yields discrete values, not only for distances, but for the orbital velocities as well.

It should be also noted that this analysis does not deal with the masses of the planets since these depend on the initial dust and gas distribution.

Equations (32) and (33) contain a cube root, which means that there are three degrees of freedom in both distance and velocity (reason for the mental picture of an onion).

\subsection{Quantized Galaxy Redshifts}

If the local group of galaxies is considered as a nonlinear closed energy system, then one would expect to see the effects of a period doubling process on it. W.G. Tifft of the University of Arizona discovered that the redshift difference of galaxy pairs grouped around $v \approx 72 \mathrm{~km} / \mathrm{s}$ and $\nu \approx 36 \mathrm{~km} / \mathrm{s}$ (most pronounced), if redshift is interpreted as velocity. He further noticed that the redshifts of individual galaxies grouped in the same way $[13,14,15]$.

The values reflect the period doubling mechanism, since

$$
\frac{72 \mathrm{~km} / \mathrm{s}}{\mathrm{c}}=2^{-12.03}
$$

and

$$
\frac{36 \mathrm{~km} / \mathrm{s}}{\mathrm{c}}=2^{-13.03}
$$

Tifft has later measured (using the $21 \mathrm{~cm}$ Hydrogen line) many additional redshifts. All fit the doubling scheme (also known as the ninth-root Lehto-Tifft rule including the redshift variability).

The redshift quantization in the local group was confirmed by Napier and Guthrie [16], but the phenomenon is largely disputed by the mainstream cosmologists.

\section{Period Doubling in $1 / \mathbf{r}-$ Nonlinear Systems}

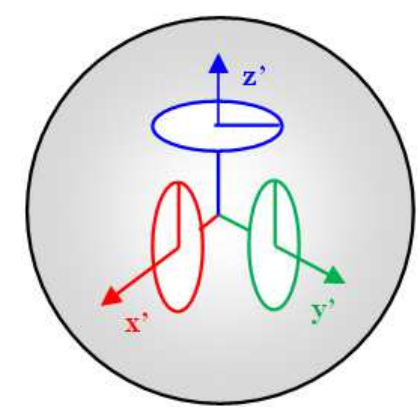

Figure 7. A closed system with internal degrees of freedom.
The gray sphere in Fig. 7 describes a system, which has three internal degrees of freedom, and which can be described by rotation and vibration about the internal $\left(x^{\prime}, y^{\prime}\right.$, $\left.z^{\prime}\right)$-axes. The system can freely move in the normal external $(x, y, z)$-space.

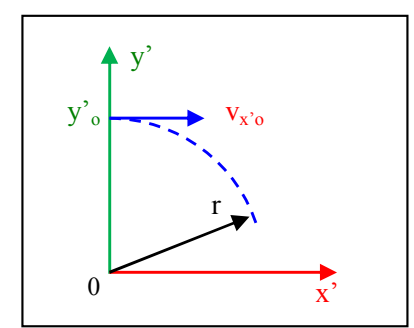

Figure 8. Initial setting for one degree of freedom.

Nonlinearity is required for the period doubling process to occur in the system. Our interest is in the gravitational and Coulomb potentials (and their gradients), which are naturally nonlinear. The well known $-1 / r$ potential leads to the $1 / r^{2}$ force and Kepler's third law, which indicates that $r^{3}=\tau^{2}$ (omitting the constant of proportionality). Solving for $r$ and taking the second derivative with respect to period $\tau$ one obtains

$$
\frac{d^{2} r}{d \tau^{2}}=-\frac{2}{9} \tau^{-\frac{4}{3}}
$$

By again applying Kepler's third law, (36) can be written in the form

$$
\frac{d^{2} r}{d \tau^{2}}=-\frac{2}{9} \cdot \frac{1}{\tau^{2}} r
$$

Eq. (37) is formally an equation of motion in terms of period of an oscillator, whose spring constant is inversely proportional to the period squared. This means that the amplitude grows and the oscillations slow down with an increasing period. It should be noted that (37) does not describe a real motion, since this would require using time $t$ instead of period $\tau$.

The oscillatory solution of (37) would result in positive and negative values of $r$ and therefore we rewrite (35) as follows:

$$
\begin{aligned}
& \frac{d^{2} x^{\prime}}{d \tau^{2}}=-\frac{a}{\tau^{2}} x^{\prime} \\
& \frac{d^{2} y^{\prime}}{d \tau^{2}}=-\frac{a}{\tau^{2}} y^{\prime}
\end{aligned}
$$

Equations (38) and (39) describe oscillations in the internal $x$ ' and $y$ ' directions and a 90 degree phase difference between them will produce a behavior analogous to circular motion about $z$ '. We are using constant $a$ in (38) and (39), because we are interested in a solution which doubles the volume $r^{3}$.

We shall now proceed with solving (38) and (39) simultaneously for an initial setting shown in Figure 8 and 
reconstruct the radius using the Pythagorean theorem $r_{z}=\operatorname{sqrt}\left(x^{, 2}+y^{, 2}\right)$.

The 90 degree phase difference between the $x$ '- and $y$ '-rotations is taken care of by choosing proper initial values. The test object is initially located at $\left(0, y^{\prime}{ }_{o}=1\right)$ and given an initial velocity $\left(v_{x^{\prime} o}, v_{y^{\prime}}=0\right)$. Equations are solved starting from an initial period $\tau=1$. The initial $x$ '-component of velocity is $v_{x^{\prime} o}=\operatorname{sqrt}(a)$ and the value of $a$ is 46.47014 for volumetric (3-d) doubling.

In principle, volumetric doubling in 4 dimensions does not differ from the 3-dimensional doubling. In this case the value of the constant $a$ is 82.4 .

The dimensionless constant $a$ is not a physical spring constant. Equation (37) shows however, that the $1 / r$ nonlinearity can produce the observed volumetric doubling, when proper initial conditions are satisfied.

Figure 9 is a plot of a simultaneous numerical solution to (38) and (39) showing radius $r$ as a function of the period $\tau$. One can see that there are plateaus, where the radius remains constant over a range of periods. The ratio of adjacent radii (plateau values) is the cube root of two, as required by the observed distribution in Figure 1 of the fractional parts.

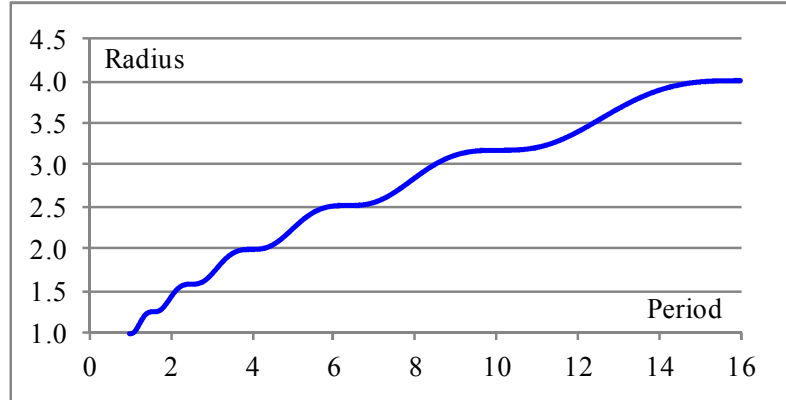

Figure 9. Radius as a function of period.

Figure 10 shows volume $r_{z}{ }^{3}$ as a function of period squared. With the parameters given the plateaus of constant volume occur at doubling volumes (1, 2, 4, 8, 16, 32 etc.).

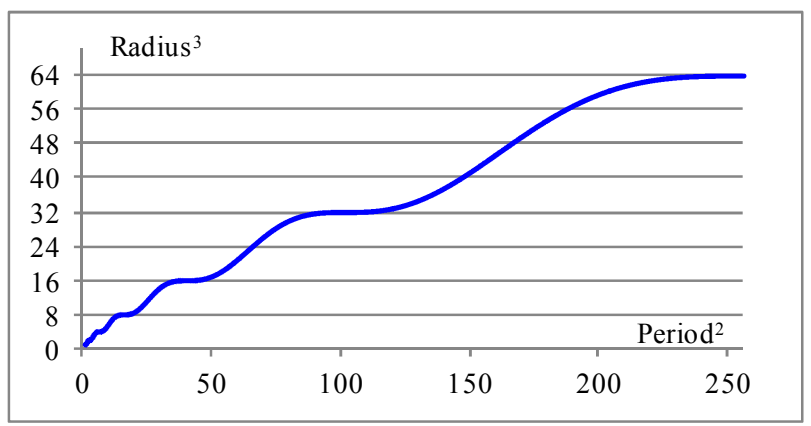

Figure 10. Radius cubed a as function of period

Corresponding calculation for $r_{x}$, and $r_{y}$, can be carried out (rotation about $x$ '- and $y^{\prime}$-axes). Here we limit ourselves to identical rotations about the three axes and the volume is therefore represented by $V=r_{z}{ }^{3}$ (cube) rather than by $V=r_{x}, r_{y}, r_{z}$, (right parallelepiped). We could also have written the volume in terms of period or energy. Similar reasoning applies to four degrees of freedom.

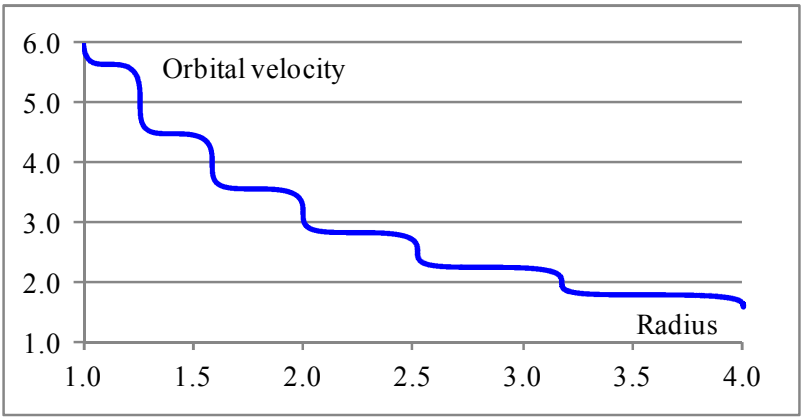

Figure 11. Orbital velocity as a function of radius.

Figure 11 shows the internal 'orbital velocity' $\nu=2 \pi r_{z}, \tau$ for the rotational degree of freedom as a function of the radius. It can be seen that the orbital velocity is quantized and the ratio of adjacent values is the cube root of two.

The ratio of radii at the transition points is likewise a cube root of two and the velocity remains constant over a range of radii. Note that the constant velocity plateaus become longer and longer with growing radius.

Equation (37) describes the behavior of an unperturbed system. Systems in real environments are seldom fully isolated but under continuous influence of perturbations.

A damped analytical solution to (37) of the type $r=\sin \left[\operatorname{const} \cdot \ln \left(\tau / \tau_{o}\right)\right]$ exists. This is not a physical solution, however, since the radius $r$ does not grow with increasing period $\tau$. Small perturbations can be taken into account by adding attenuation (or amplification) in Eq. (37) such that the constant of attenuation is inversely proportional to period $(b / \tau$ in $(40))$ :

$$
\frac{d^{2} r}{d \tau^{2}}=-\frac{a}{\tau^{2}} r-\frac{b}{\tau} \frac{d r}{d \tau}
$$

\section{Discussion}

Period doubling is a general property of nonlinear dynamical systems. It can be rather safely stated that nothing in nature is truly linear, and therefore it is tempting to analyze different physical systems for discovering the possibly hidden occurrence of the period doubling phenomenon.

The early study [10] already gave some results pointing to the intrinsic degrees of freedom and related period doubling. However, it was not understood at that time that the mass-energy and electromagnetic energy systems are different and independent. It became later [11, 12] evident that the mass-systems have three intrinsic degrees of freedom (3-d), whereas the Coulombic-systems possess four degrees of freedom (4-d). This leads to non-polar mass and polar electricity in a natural way. In particle analysis it is convenient to combine the 3-d and 4-d systems into one equation (14), which also takes the rotational and vibrational modes into account.

The existence of the intrinsic degrees of freedom cannot be 
directly seen from the observational data, because a measurement returns the geometric mean of e.g. the energy content of each degree of freedom.

The great accuracy of the calculated values for the elementary charge, the fine structure constant, the magnetic moments of the electron, proton and neutron, indicates that the period doubling process is precise even after a large number of period doublings.

The Planck scale values of different physical quantities can be used as reference values. It means that there is a direct connection between the natural constants and properties of matter.

We can also say that we would not exist without the long term stability of the basic constituents of matter, i.e. electrons and protons. It is not much of a surprise then that the number of period doublings for the basic constituents and the related phenomena is in accordance with the theoretical stability condition (3).

Equation (21) suggests that the proton alone is superstable, not the proton-antiproton pair. It is therefore tempting to think that a proton is actually a positron in disguise, because the electron-positron pair is superstable. If so, we do not need to search for the possible anti-universes.

What may be surprising is the close relation between the electron-positron pair, CBR and the $21 \mathrm{~cm}$ Hydrogen line. The origin of the CBR may not be the 'Big Bang' at all.

The initial gas and dust cloud around the Sun seems to have undergone period doubling, since the material has accumulated in corresponding orbits. The orbital velocities of the planets are determined by consequent $N$-numbers in (32) with one exception. There is an allowed unoccupied orbit next to and outside Jupiter's orbit. Figure 5 shows that Jupiter has moved towards the empty orbit as if the missing planet had pulled it there.

If the local group of galaxies is considered as a local energy system, like the Solar system, one would expect to find an energy related period doubling phenomenon. The redshift quantization seems to be one.

The behavior of nonlinear dynamical systems is normally analyzed using space-time coordinates. In this study space-period is used instead, because we are interested in the period doubling phenomenon.

A second order differential equation is derived for $1 / r$-nonlinear systems using Kepler's law. The solution shows that 3-d and 4-d period doubling is possible given proper parameter values in (38) and (39).

\section{Acknowledgements}

I would like to express my great appreciation to the members of the Physics Foundations Society, Dr. Tuomo Suntola, Dr. Heikki Sipilä, Dr. Tarja Kallio-Tamminen, Dr. Mervi Hyvönen-Dabek and Dr. Jan Dabek, for their valuable and constructive suggestions and help during the preparation of the manuscript.

\section{References}

[1] Feigenbaum M. J., "Quantitative Universality for a Class of Non-Linear Transformations." J. Stat. Phys. 19, 25-52, 1978

[2] Feigenbaum M. J., "Universal Behavior in Nonlinear Systems", Chaos and Universality, Nordita reprint selection, November 1981

[3] http://en.wikipedia.org/wiki/Attractor (10 Sep. 2014)

[4] Simpson T. B., Liu J. M., Gavrielides A., Kovanis V. and Alsing P. M., "Period-doubling route to chaos in a semiconductor laser subject to optical injection", Appl. Phys. Lett. 64, 3539, 1994

[5] Cheung P. Y. and Wong A. Y., "Chaotic behavior and period doubling in plasmas", Phys. Rev. Lett. 59, 551, 3 August 1987

[6] Batygin K., Morbidelli A., "Onset of Secular Chaos in Planetary Systems: Period Doubling \& Strange Attractors", arXiv: 1106.2590

[7] Moskalik P. and Buchler J. R., "Resonances and period doubling in the pulsations of stellar models", Astrophysical Journal, Part 1, vol. 355, p. 590-601, June 1, 1990

[8] Gleick James, "Chaos: Making a New Science", Viking Penguin, 1987

[9] Baryshev Y. and Teerikorpi P., "Discovery of Cosmic Fractals", World Scientific Co Pte Ltd, 2003

[10] Lehto A., "On (3+3)-dimensional discrete space-time", University of Helsinki, Report Series in Physics HU-P-236, 1984

[11] Lehto A., "Periodic time and the stationary properties of matter". Chin. J. Phys. 28 (3), 215-235, 1990

[12] Lehto A., "On the Planck Scale and Properties of Matter", Nonlinear Dynamics, 55, 3, 279-298, February 2009

[13] Tifft W. G., "Discrete states of redshift and galaxy dynamics. I - Internal motions in single galaxies", Astrophysical Journal 206: 38-56, 1976

[14] Tifft W. G., "Discrete states of redshift and galaxy dynamics. II - Systems of galaxies". Astrophysical Journal 211: 31-46, 1977

[15] Tifft W .G., "Redshift Quantization - A Review", Astrophysics and Space Science 227 (1-2), 1995

[16] Napier W.M. and Guthrie B.N.G., "Testing for Quantized Redshifts. I. Project", Astrophysics and Space Science, 244, 57-63, 1996 